\title{
THE NEUTRIX CONVOLUTION PRODUCT $\left(X_{-}^{\lambda} \ln ^{R} X_{-}\right) \circledast\left(X_{+}^{\mu} \ln ^{S} X_{+}\right)$
}

Summary. The existence of the neutrix convolution product $f \circledast g$ as well as the explicit formulae are proved for the distributions $f(x)=x_{-}^{\lambda} \ln ^{r} x_{-}$and $g(x)=$ $x_{+}^{\mu} \ln ^{s} x_{+}$, where $r, s \in\{0\} \cup \mathbf{N}$ and $\lambda, \mu$ are real numbers such that $\lambda, \mu \notin-\mathbf{N}$ and $\lambda+\mu \notin \mathbf{Z}$, and for some related pairs of distributions. The theorems of the paper generalize earlier results proved in the case $r=s=0$ and $\lambda, \mu, \lambda+\mu \notin \mathbf{Z}$.

\section{SPLOT $\left(X_{-}^{\lambda} \ln ^{R} X_{-}\right) \circledast\left(X_{+}^{\mu} \ln ^{S} X_{+}\right)$W SENSIE NEUTRIKSU}

Streszczenie. W pracy dowodzi się istnienia splotu $f \circledast g$ w sensie neutriksu i znajduje jego wartość dla dystrybucji $f(x)=x_{-}^{\lambda} \ln ^{r} x_{-}$i $g(x)=x_{+}^{\mu} \ln ^{s} x_{+}$, gdzie $r, s \in\{0\} \cup \mathbf{N}$, a $\lambda, \mu$ są liczbami rzeczywistymi, takimi ze $\lambda, \mu \notin-\mathbf{N} ; \lambda+\mu \notin \mathbf{Z}$, a także dla pewnych innych par dystrybucji. Twierdzenia podane w pracy uogólniają wcześniejsze wyniki otrzymane w przypadku, gdy $r=s=0$ oraz $\lambda, \mu, \lambda+\mu \notin \mathbf{Z}$.

In the following we denote by $\mathbf{Z}$ the set of all integers, by $\mathbf{N}$ the set of all positive integers, by $-\mathbf{N}$ the set of all negative integers, by $\mathbf{N}_{0}$ the set of all nonnegative integers, by $-\mathbf{N}_{0}$ the set of all nonpositive integers and by $\mathbf{R}$ the set of all reals.

Moreover, we let $\mathcal{D}$ be the space of infinitely differentiable functions on $\mathbf{R}$ with compact support and $\mathcal{D}^{\prime}$ be the space of distributions on $\mathbf{R}$, i.e. linear continuous functionals defined on $\mathcal{D}$ endowed with an appropriate topology (see e.g. [7]). 
Definition 1. Suppose that $f$ and $g$ are distributions in $\mathcal{D}^{\prime}$ whose supports $A$ and $B$ satisfy the following condition of compatibility: for every compact set $K \subset \mathbf{R}$, the set $(K-A) \cap B$ is compact in $\mathbf{R}$. Then the convolution product $f * g$ in $\mathcal{D}^{\prime}$ is defined by the formula:

$$
\langle(f * g)(x), \phi\rangle=\langle f(x),\langle g(y), \phi(x+y)\rangle\rangle
$$

for arbitrary $\phi$ in $\mathcal{D}(c f .[7])$.

Obviously, the above definition embraces the two following particular cases of compatible supports:

(a) either $A$ or $B$ is bounded;

(b) $A$ and $B$ are bounded on the same side.

It follows from Definition 1 that if the convolution product $f * g$ exists, then also the convolution products $g * f, f * g^{\prime}, f^{\prime} * g$ and $f^{\vee} * g^{\vee}$ exist and

$$
\begin{aligned}
f * g & =g * f, \\
(f * g)^{\prime} & =f * g^{\prime}=f^{\prime} * g, \\
(f \circledast g)^{\vee} & =f^{\vee} \circledast g^{\vee},
\end{aligned}
$$

where ${ }^{\vee}$ is the operation of replacing variable $x$ by $-x$, defined formally as follows: $\phi^{\vee}(x):=$ $\phi(-x)$ for $\phi \in \mathcal{D}, x \in \mathbf{R}$ and $\left\langle f^{\vee}, \phi\right\rangle:=\left\langle f, \phi^{\vee}\right\rangle$ for $f \in \mathcal{D}^{\prime}, \phi \in \mathcal{D}$ (see [6]).

There exist in the literature various general, without any restrictions on the supports, definitions of the convolution product of distributions (cf. [6]), but for many pairs of distributions such convolution products do not exist.

In [2] the neutrix convolution product was defined so that it exists for a considerably larger class of pairs of distributions. In order to recall the definition of the neutrix convolution product we first of all let $\tau$ be a fixed function in $\mathcal{D}$ satisfying the following properties:

(i) $\tau(x)=\tau(-x)$,

(ii) $0 \leq \tau(x) \leq 1$,

(iii) $\tau(x)=1$ for $|x| \leq \frac{1}{2}$,

(iv) $\tau(x)=0$ for $|x| \geq 1$.

Next we define the sequence $\left\{\tau_{n}\right\}$ of functions setting

$$
T_{n}(x)= \begin{cases}1 & \text { if }|x| \leq n \\ \tau\left(n^{n} x-n^{n+1}\right) & \text { if } x>n \\ \tau\left(n^{n} x+n^{n+1}\right) & \text { if } x<-n,\end{cases}
$$

for $n \in \mathbf{N}$. 
Throughout the paper, given a distribution $f$, by $f_{n}$ we denote the distributions of the form

$$
f_{n}:=f \tau_{n}
$$

for $n \in \mathbf{N}$.

The notion of a neutrix which allows the extension of limits of numerical sequences was introduced by van der Corput in [1] and is based on a suitably chosen set of negligible functions.

As in [2], we adopt in this paper the following definition of negligible functions:

Definition 2. The set of negligible functions of the neutrix $N$ with the domain $N^{\prime}=$ $\mathbf{N}$ and the range $N^{\prime \prime}=\mathbf{R}$ consists of all finite linear sums of the functions

$$
n^{\lambda} \ln ^{r-1} n, \ln ^{r} n \quad(\lambda>0, r \in \mathbf{N})
$$

and all functions which converge to zero in the usual sense as $n$ tends to infinity.

Recall now the definition of the neutrix convolution product given in [2].

Definition 3. The neutrix convolution product $f \circledast g$ of two distributions $f$ and $g$ in $\mathcal{D}^{\prime}$ is defined as the neutrix limit of the sequence $\left\{f_{n} * g\right\}$, provided that the limit $h \in \mathcal{D}^{\prime}$ exists in the sense that

$$
\underset{n \rightarrow \infty}{N-\lim _{n}}\left\langle f_{n} * g, \phi\right\rangle=\langle h, \phi\rangle
$$

for all $\phi$ in $\mathcal{D}$, where $N$ is the neutrix described in Definition 2.

Note that in this definition the convolution product $f_{n} * g$ is meant in the sense of Definition 1 (the distributions $f_{n}$ have bounded support since the support of $\tau_{n}$ is contained in the interval $\left.\left[-n-n^{-n}, n+n^{-n}\right]\right)$ and that the distribution $h$ in Definition 3 is unique.

The following theorem was proved in [6] and shows that Definition 3 is an extension of Definition 1.

Theorem 1. Let $f$ and $g$ be distributions with compatible supports. Then the neutrix convolution product $f \circledast g$ exists and

$$
f \circledast g=f * g \text {. }
$$


The neutrix convolution product has the following important properties, analogous to the first of equations in (2) and to (3) (see [2] and [6]):

Theorem 2. Let $f$ and $g$ be distributions in $\mathcal{D}^{\prime}$ and suppose that the neutrix convolution product $f \circledast g$ exists. Then the neutrix convolution products $f \circledast g^{\prime}$ and $f^{\vee} \circledast g^{\vee}$ exist and

$$
\begin{aligned}
(f \circledast g)^{\prime} & =f \circledast g^{\prime} ; \\
(f \circledast g)^{\vee} & =f^{\vee} \circledast g^{\vee} .
\end{aligned}
$$

Note however that equation (1) does not necessarily hold for the neutrix convolution product and that $(f \circledast g)^{\prime}$ is not necessarily equal to $f^{\prime} \circledast g$.

In [2] the following result was obtained:

Theorem 3. The neutrix convolution product $x_{-}^{\lambda} \circledast x_{+}^{s}$ exists and

$$
x_{-}^{\lambda} \circledast x_{+}^{s}=(-1)^{s+1} B(\lambda+1, s+1) x_{-}^{\lambda+s+1}
$$

for $\lambda \in(-1, \infty)$ and $s \in \mathbf{N}_{0}$, where $B$ denotes the beta function.

Later, the following two theorems were proved in [3] and [4], respectively:

Theorem 4. The neutrix convolution product $x_{-}^{\lambda} \circledast x_{+}^{s}$ exists and satisfies equation (4) for $\lambda \in(-\infty,-1] \backslash(-\mathbf{N})$ and $s \in \mathbf{N}_{0}$.

Theorem 5. The neutrix convolution product $x_{-}^{s} \odot x_{+}^{\lambda}$ exists and

$$
x_{-}^{s} \circledast x_{+}^{\lambda}=(-1)^{s+1} B(\lambda+1, s+1) x_{+}^{\lambda+s+1}
$$

for $\lambda \in \mathbf{R} \backslash \mathbf{Z}$ and $s \in \mathbf{N}_{0}$.

The next theorem was proved in [5].

Theorem 6. The neutrix convolution product $x_{-}^{\lambda} \circledast x_{+}^{\mu}$ exists and

$$
x_{-}^{\lambda} \circledast x_{+}^{\mu}=B(-\lambda-\mu-1, \mu+1) x_{-}^{\lambda+\mu+1}+B(-\lambda-\mu-1, \lambda+1) x_{+}^{\lambda+\mu+1},
$$

for $\lambda, \mu \in \mathbf{R}$ such that $\lambda, \mu, \lambda+\mu \notin \mathbf{Z}$. 
In the following, we are going to generalize the last theorem by proving the existence of the convolution products of the form $\left(x_{-}^{\lambda} \ln ^{r} x_{-}\right) \circledast\left(x_{+}^{\mu} \ln ^{s} x_{+}\right)$for all $r, s \in \mathbf{N}_{0}$ and $\lambda, \mu$ such that $\lambda, \mu \notin-\mathbf{N}$ and $\lambda+\mu \notin \mathbf{Z}$. It appears that these convolution products may be expressed in a concise form as the respective distributional derivatives with respect to $\lambda$ and $\mu$ of the right hand side of (5). For this aim we need some auxiliary results on the beta function.

It was proved in [9] that

$$
B_{r, s}(\lambda, \mu):=D_{\lambda}^{r} D_{\mu}^{s} B(\lambda, \mu)=\underset{n \rightarrow \infty}{\mathrm{N}}-\lim _{1 / n} \int^{1-1 / n} t^{\lambda-1} \ln ^{r} t(1-t)^{\mu-1} \ln ^{s}(1-t) d t
$$

for $r, s \in \mathbf{N}_{0}$ and $\lambda, \mu \notin-\mathbf{N}_{0}$, where

$$
D_{\lambda}^{r}:=\frac{\partial^{r}}{\partial \lambda^{r}}, \quad D_{\mu}^{s}:=\frac{\partial^{s}}{\partial \mu^{s}} .
$$

In particular, if $\mu>0$ and $\lambda \notin-\mathbf{N}_{0}$, the above expression can be replaced by

$$
B_{r, s}(\lambda, \mu)=\underset{n \rightarrow \infty}{N}-\lim _{1 / n} \int^{1} t^{\lambda-1} \ln ^{r} t(1-t)^{\mu-1} \ln ^{s}(1-t) d t .
$$

In the lemma below $\left(\alpha_{n}\right)$ is the sequence of positive numbers tending to 0 , given by one of the formulae:

(a) $\alpha_{n}=1 / n$ for $n \in \mathbf{N}$;

(b) $\alpha_{n}=x / n$ for $n \in \mathbf{N}$;

(c) $\alpha_{n}=x /(x+n)$ for $n \in \mathbf{N}$.

Lemma. If $x>0$, then

$$
B_{r, s}(\lambda, \mu)=\mathrm{N}-\lim _{n \rightarrow \infty} \int_{\alpha_{n}}^{1} t^{\lambda-1} \ln ^{r} t(1-t)^{\mu-1} \ln ^{s}(1-t) d t
$$

for $r, s \in \mathbf{N}_{0}, \mu \in(0, \infty)$ and $\lambda \in \mathbf{R} \backslash\left(-\mathbf{N}_{0}\right)$, where $\left(\alpha_{n}\right)$ is any of the three numerical sequences given by formulas $(a)-(c)$ above.

Proof. Choose a positive integer $p$ such that $p+\lambda>0$ and let $\sum_{i=0}^{p-1} a_{i} t^{i}$ be the sum of the first $p$ terms in the Taylor expansion of $(1-t)^{\mu-1} \ln ^{9}(1-t)$.

We shall prove first that

$$
\underset{n \rightarrow \infty}{N-\lim } \int_{\alpha_{n}}^{1} t^{\lambda+i-1} \ln ^{r} t d t=\frac{(-1)^{r} r !}{(\lambda+i)^{r+1}}
$$

for $r \in \mathbf{N}_{0}$ in all cases $(a)-(c)$, defined above. 
Since $\lambda+i \neq 0$, we have

$$
\int_{\alpha}^{1} t^{\lambda+i-1} d t=\frac{1-\alpha^{\lambda+i}}{\lambda+i}
$$

and further, if $r \in \mathbf{N}$,

$$
\int_{\alpha}^{1} t^{\lambda+i-1} \ln ^{r} t d t=-\frac{\alpha^{\lambda+i} \ln ^{r+1} \alpha}{\lambda+i}-\frac{r}{\lambda+i} \int_{\alpha}^{1} t^{\lambda+i-1} \ln ^{r-1} t d t
$$

Replacing $\alpha$ in (9) and (10) by $\alpha_{n}=x /(x+n)$, we have

$$
\int_{\alpha_{n}}^{1} t^{\lambda+i-1} d t=\frac{1-n^{-\lambda-i} x^{\lambda+i}(1+x / n)^{-\lambda-i}}{\lambda+i}
$$

and

$$
\int_{\alpha_{n}}^{1} t^{\lambda+i-1} \ln ^{r} t d t=-\frac{1}{(\lambda+i)}\left[\alpha_{n}^{\lambda+i}(\ln x-\ln (x+n))^{r}+r \int_{\alpha_{n}}^{1} t^{\lambda+i-1} \ln ^{r-1} t d t\right],
$$

since $\lambda$ is not an integer. It follows by induction that

$$
\mathrm{N}-\lim _{n \rightarrow \infty} \int_{x /(x+n)}^{1} t^{\lambda+i-1} \ln ^{r+1} t d t=\frac{(-1)^{r} r !}{(\lambda+i)^{r+1}}
$$

for $r \in \mathbf{N}$, i.e. (8) follows in case (c).

In a similar way, one can check that (8) holds true in cases $(a)$ and $(b)$.

Since $p+\lambda>0$, the integral

$$
I:=\int_{0}^{1} t^{\lambda-1} \ln ^{r} t\left[(1-t)^{\mu-1} \ln ^{s}(1-t)-\sum_{i=0}^{p-1} a_{i} t^{i}\right] d t
$$

exists. Hence, in view of (6) and (8),

$$
\begin{aligned}
& B_{r, s}(\lambda, \mu)=\underset{n \rightarrow \infty}{\mathrm{N}-\lim _{1 / n}} \int_{1}^{1} t^{\lambda-1} \ln ^{r} t(1-t)^{\mu-1} \ln ^{9}(1-t) d t \\
& =I+\sum_{i=0}^{p-1} a_{i}\left[\mathrm{~N}-\lim _{n \rightarrow \infty} \int_{1 / n}^{1} t^{\lambda+i-1} \ln ^{r} t d t\right] \\
& =I+\sum_{i=0}^{p-1} a_{i}\left[\mathrm{~N}-\lim _{n \rightarrow \infty} \int_{\alpha_{n}}^{1} t^{\lambda+i-1} \ln ^{r} t d t\right] \\
& =N-\lim _{n \rightarrow \infty} \int_{\alpha_{n}}^{1} t^{\lambda-1} \ln ^{\gamma} t(1-t)^{\mu-1} \ln ^{s}(1-t) d t
\end{aligned}
$$

for the sequences $\left(\alpha_{n}\right)$ of the form $(b)$ and $(c)$.

Consequently, equation (7) follows. 
Theorem 7. The neutrix convolution product $\left(x_{-}^{\lambda} \ln ^{r} x_{-}\right) \circledast\left(x_{+}^{\mu} \ln ^{s} x_{+}\right)$exists and

$$
\begin{aligned}
& \left(x_{-}^{\lambda} \operatorname{In}^{r} x_{-}\right) \circledast\left(x_{+}^{\mu} \ln ^{s} x_{+}\right) \\
& =D_{\lambda}^{r} D_{\mu}^{s}\left[B(-\lambda-\mu-1, \mu+1) x_{-}^{\lambda+\mu+1}+B(-\lambda-\mu-1, \lambda+1) x_{+}^{\lambda+\mu+1}\right]
\end{aligned}
$$

for $r, s \in \mathbf{N}_{0}$ and $\lambda, \mu \in \mathbf{R} \backslash(-\mathbf{N})$ such that $\lambda+\mu \notin \mathbf{Z}$.

Proof. The proof will consist of three parts, depending on the values of $\lambda$ and $\mu$. In all three parts we assume that $r, s \in \mathbf{N}_{0}$.

Part I: $\quad \lambda, \mu>-1 ; \lambda+\mu \notin\{-1\} \cup \mathbf{N}_{0}$.

First notice that in our case $x_{-}^{\lambda} \ln ^{r} x_{-}$and $x_{+}^{\mu} \ln ^{s} x_{+}$are locally integrable functions. Put

$$
\left(x_{-}^{\lambda} \ln ^{r} x_{-}\right)_{n}:=\left(x_{-}^{\lambda} \ln ^{r} x_{-}\right) \tau_{n}(x) .
$$

Then the convolution product $\left(x_{-}^{\lambda} \ln ^{r} x_{-}\right)_{n} *\left(x_{+}^{\mu} \ln ^{s} x_{+}\right)$exists both in the sense of Definition 1 and in the classical sense and we have

$$
\begin{aligned}
& \left(x_{-}^{\lambda} \ln ^{r} x_{-}\right)_{n} *\left(x_{+}^{\mu} \ln ^{s} x_{+}\right) \\
& =\int_{-\infty}^{\infty} y_{-}^{\lambda} \ln ^{r} y_{-} \tau_{n}(y)(x-y)_{+}^{\mu} \ln ^{s}(x-y)_{+} d y=I_{1}^{n}+I_{2}^{n},
\end{aligned}
$$

where

$$
\begin{aligned}
& I_{1}^{n}:=\int_{-n}^{0}(-y)^{\lambda} \ln ^{r}(-y)(x-y)_{+}^{\mu} \ln ^{s}(x-y)_{+} d y \\
& I_{2}^{n}:=\int_{-n-n-n}^{-n}(-y)^{\lambda} \ln ^{r}(-y) \tau_{n}(y)(x-y)_{+}^{\mu} \ln ^{s}(x-y)_{+} d y
\end{aligned}
$$

In the case $x<0$, we substitute in the integral in (13) $y=x t^{-1}$ and obtain

$$
\begin{aligned}
& I_{1}^{n}=\int_{-n}^{x}(-y)^{\lambda} \ln ^{r}(-y)(x-y)^{\mu} \ln ^{s}(x-y) d y \\
& =(-x)^{\lambda+\mu+1} \int_{-x / r l}^{1} t^{-\lambda-\mu-2}[\ln (-x)-\ln t]^{r}(1-t)^{\mu}[\ln (-x)+\ln (1-t)-\ln t]^{s} d t \\
& =\sum_{i=0}^{r} \sum_{k=0}^{s} \sum_{j=0}^{k} c_{i, j, k} I_{i, j, k}^{n}(-x)^{\lambda+\mu+1} \ln ^{r+s-i-k}(-x),
\end{aligned}
$$

where

$$
c_{i, j, k}:=(-1)^{i+j}\left(\begin{array}{l}
r \\
i
\end{array}\right)\left(\begin{array}{l}
s \\
k
\end{array}\right)\left(\begin{array}{l}
k \\
j
\end{array}\right) ; \quad I_{i, j, k}^{n}:=\int_{-x / n}^{1} t^{-\lambda-\mu-2} \ln ^{i+j} t(1-t)^{\mu} \ln ^{k-j}(1-t) d t
$$

for the respective integers $i, j, k$. 
Since $-x>0$, it follows from the lemma that

$$
\underset{n \rightarrow \infty}{\mathrm{N}}-\lim _{i, j, k} I_{i+j, k-j}^{n}(-\lambda-\mu-1, \mu+1)
$$

for the respective $i, j, k$.

On the other hand, using the formula $x_{+}^{\lambda} \ln ^{p} x_{+}=D_{\lambda}^{p} x_{+}^{\lambda}$ for $p \in \mathbf{N}$ (see [8]) and the equations:

$$
\begin{aligned}
& D_{\lambda} B_{l, m}(-\lambda-\mu-1, \mu+1)=-B_{l+1, m}(-\lambda-\mu-1, \mu+1) ; \\
& D_{\mu} B_{l, m}(-\lambda-\mu-1, \mu+1)= \\
& \quad=-B_{l+1, m}(-\lambda-\mu-1, \mu+1)+B_{l, m+1}(-\lambda-\mu-1, \mu+1),
\end{aligned}
$$

valid for any $l, m \in \mathbf{N}$, one can prove by induction with respect to $r+s$ that

$$
\begin{aligned}
& D_{\lambda}^{r} D_{\mu}^{s}\left[B(-\lambda-\mu-1, \mu+1) x_{-}^{\lambda+\mu+1}\right]= \\
& =\sum_{i=0}^{r} \sum_{k=0}^{s} \sum_{j=0}^{k} c_{i, j, k} B_{i+j, k-j}(-\lambda-\mu-1, \mu+1) x_{-}^{\lambda+\mu+1} \ln ^{r+s-i-k} x_{-} .
\end{aligned}
$$

Combining (15), (16) and (17), we get

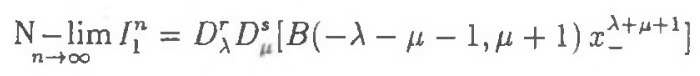

in the case $x<0$.

If $x>0$, we use the substitution $y=x\left(1-t^{-1}\right)$ in the integral in (13) and then

$$
\begin{aligned}
& I_{1}^{n}=\int_{-n}^{0}(-y)^{\lambda} \ln ^{r}(-y)(x-y)^{\mu} \ln ^{s}(x-y) d y \\
& =x^{\lambda+\mu+1} \int_{x /(x+\pi)}^{1} t^{-\lambda-\mu-2}[\ln x+\ln (1-t)-\ln t]^{r}(1-t)^{\lambda}[\ln x-\ln t]^{s} d t \\
& =\sum_{i=0}^{s} \sum_{k=0}^{r} \sum_{j=0}^{k} c_{i, j, k} J_{i, j, k}^{n} x^{\lambda+\mu+1} \ln ^{r+s-i-k} x
\end{aligned}
$$

where

$$
J_{i, j, k}^{n}:=\int_{x /(x+n)}^{1} t^{-\lambda-\mu-2} \ln ^{i+j} t(1-t)^{\lambda} \ln ^{k-j}(1-t) d t .
$$

By the lemma,

$$
\underset{n \rightarrow \infty}{\mathrm{N}-\lim _{i, j, k}} J_{i+j, k-\jmath}^{n}(-\lambda-\mu-1, \lambda+1)
$$

for the respective integers $i, j, k$.

On the other hand, replacing $-x$ by $x$ and interchanging $\lambda$ and $\mu$ as well as $r$ and $s$ in (17), we get

$$
\begin{aligned}
& D_{\lambda}^{r} D_{\mu}^{s}\left[B(-\lambda-\mu-1, \lambda+1) x_{-}^{\lambda+\mu+1}\right]= \\
& =\sum_{i=0}^{s} \sum_{k=0}^{r} \sum_{j=0}^{k} c_{i, j, k} B_{i+j, k-j}(-\lambda-\mu-1, \lambda+1) x_{+}^{\lambda+\mu+1} \ln ^{r+s-i-k} x_{+} .
\end{aligned}
$$


Combining (19), (20) and (21), we obtain

$$
\underset{n \rightarrow \infty}{\mathrm{N}-\lim } I_{1}^{n}=D_{\lambda}^{r} D_{\mu}^{s}\left[B(-\lambda-\mu-1, \lambda+1) x_{+}^{\lambda+\mu+1}\right]
$$

in the case $x>0$.

Further, it is easily seen that

$$
I_{2}^{n}=O\left(n^{-n+\lambda+\mu} \ln ^{r+s} n\right)
$$

so

$$
\mathrm{N}-\lim _{n \rightarrow \infty} I_{2}^{n}=0 \text {. }
$$

It now follows from equations (12), (18), (22) and (23) that

$$
\begin{aligned}
& \underset{n \rightarrow \infty}{\mathrm{N}}-\lim _{n}\left(x_{-}^{\lambda} \ln ^{r} x_{-}\right)_{n} *\left(x_{+}^{\mu} \ln ^{s} x_{+}\right)= \\
& =D_{\lambda}^{r} D_{\mu}^{s}\left[B(-\lambda-\mu-1, \mu+1) x_{-}^{\lambda+\mu+1}+B(-\lambda-\mu-1, \lambda+1) x_{+}^{\lambda+\mu+1}\right],
\end{aligned}
$$

which completes the proof of Part I.

Part II: $\quad \lambda>-1 ; \quad \mu \notin-\mathbf{N} ; \quad \lambda+\mu \notin \mathbf{Z}$.

Denote $\mathbf{N}_{0}^{-}:=\emptyset, \mathbf{N}_{i}^{-}:=\{-1,-2, \ldots,-i\}$ and

$$
X_{i}:=\left\{(\lambda, \mu): \lambda>-1, \mu>-i, \mu \notin \mathbf{N}_{i-1}^{-}, \lambda+\mu \notin \mathbf{N}_{i}^{-} \cup \mathbf{N}_{0},\right\} .
$$

for $i \in \mathbf{N}$.

To prove the assertion of the theorem under the conditions of this part we will show the existence of $\left(x_{-}^{\lambda} \ln ^{r} x_{-}\right) \circledast\left(x_{+}^{\mu} \ln ^{s} x_{+}\right)$and equation (11) for arbitrary $i \in N$ and $(\lambda, \mu) \in X_{i}$ by induction with respect to $i$.

For $i=1$ our assertion is valid, in view of Part I. Fix $k \in \mathbf{N}$ and suppose that the assertion holds true for $i=k$. Now take $(\lambda, \mu) \in X_{k+1}$ and.put $\bar{\mu}:=\mu+1$. Since $(\lambda, \bar{\mu}) \in X_{k}$ and $\bar{\mu} \neq 0$, the neutrix convolution product $\left(x_{-}^{\lambda} \ln ^{r} x_{-}\right) \circledast\left(x_{+}^{\bar{\mu}} \ln ^{s} x_{+}\right)$exists for arbitrary $r, s \in \mathbf{N}_{0}$. Moreover, by Theorem 2 and induction hypothesis, the neutrix convolution product $\left(x_{-}^{\lambda} \ln ^{r} x_{-}\right) \circledast\left(x_{+}^{\bar{\mu}} \ln ^{s} x_{+}\right)^{\prime}$ exists and

$$
\begin{aligned}
& \left(x_{-}^{\lambda} \ln ^{r} x_{-}\right) \circledast\left(x_{+}^{\bar{\mu}} \ln ^{s} x_{+}\right)^{\prime}=\left(x_{-}^{\lambda} \ln ^{r} x_{-}\right) \circledast\left[\bar{\mu} x_{+}^{\mu} \ln ^{s} x_{+}+s x_{+}^{\mu} \ln ^{s-1} x_{+}\right]= \\
& =\left[\left(x_{-}^{\lambda} \ln ^{r} x_{-}\right) \circledast\left(x_{+}^{\bar{\mu}} \ln ^{s} x_{+}\right)\right]^{\prime}=D_{\lambda}^{r} D_{\mu}^{s}\left[(\lambda+\mu+2) f_{\lambda, \mu}(x)\right],
\end{aligned}
$$

for $r, s \in \mathbf{N}_{0}$, where

$$
f_{\lambda, \mu}(x):=B(\lambda-\mu-2, \mu+2) x_{-}^{\lambda+\mu+1}+B(\lambda-\mu-2, \lambda+1) x_{+}^{\lambda+\mu+1} .
$$

Notice that

$$
\begin{aligned}
-(\lambda+\mu+2) B(-\lambda-\mu-2, \mu+2) & =\bar{\mu} B(-\lambda-\mu-1, \mu+1) \\
(\lambda+\mu+2) B(-\lambda-\mu-2, \lambda+1) & =\bar{\mu} B(-\lambda-\mu-1, \lambda+1),
\end{aligned}
$$


B. Fisher, A. Kamiński, A. Kiliçman

due to the known property of the gamma function and its relation to the beta function. It follows from (25) and (26) that

$$
(\lambda+\mu+2) f_{\lambda, \mu}(x)=\bar{\mu} g_{\lambda, \mu}(x),
$$

where

$$
g_{\lambda, \mu}(x):=B(-\lambda-\mu-1, \mu+1) x_{-}^{\lambda+\mu+1}+B(-\lambda-\mu-1, \lambda+1) x_{+}^{\lambda+\mu+1} .
$$

By (27),

$$
\begin{aligned}
& D_{\lambda}^{r} D_{\mu}^{s}\left[(\lambda+\mu+2) f_{\lambda, \mu}(x)\right]=D_{\lambda}^{r} D_{\mu}^{s-1} D_{\mu}^{1}\left[(\mu+1) g_{\lambda^{\prime} \mu}(x)\right]= \\
& =D_{\lambda}^{r} D_{\mu}^{s-1}\left[(\mu+1) D_{\mu}^{1} g_{\lambda, \mu}(x)\right]+D_{\lambda}^{r} D_{\mu}^{s-1} g_{\lambda, \mu}(x)= \\
& =D_{\lambda}^{r} D_{\mu}^{s-2}\left[(\mu+1) D_{\mu}^{2} g_{\lambda_{, \mu}}(x)\right]+2 D_{\lambda}^{r} D_{\mu}^{s-1} g_{\lambda, \mu}(x) .
\end{aligned}
$$

and, by induction,

$$
\begin{aligned}
& D_{\lambda}^{r} D_{\mu}^{s}\left[(\lambda+\mu+2) f_{\lambda, \mu}(x)\right]= \\
& =D_{\lambda}^{r}\left[(\mu+1) D_{\mu}^{s} g_{\lambda, \mu}(x)\right]+s D_{\lambda}^{r} D_{\mu}^{s-1} g_{\lambda, \mu}(x) .
\end{aligned}
$$

Consequently, by (24),

$$
\begin{aligned}
& \left(x_{-}^{\lambda} \ln ^{r} x_{-}\right) \circledast\left[\bar{\mu} x_{+}^{\mu} \ln ^{s} x_{+}+s x_{+}^{\mu} \ln ^{s-1} x_{+}\right] \\
& =\bar{\mu} D_{\lambda}^{r} D_{\mu}^{s} g_{\lambda, \mu}(x)+s D_{\lambda}^{r} D_{\mu}^{s-1} g_{\lambda, \mu}(x)
\end{aligned}
$$

for $r, s \in \mathbf{N}_{0}$.

Taking $s=0$, we see that $\left(x_{-}^{\lambda} \ln ^{r} x_{-}\right) \circledast x_{+}^{\mu}$ exists and, since $\bar{\mu} \neq 0$,

$$
\left(x_{-}^{\lambda} \ln ^{r} x_{-}\right) \circledast x_{+}^{\mu}=D_{\lambda}^{r} g_{\lambda, \mu}(x),
$$

i.e. (11) holds for $s=0$.

Assume that $\left(x_{-}^{\lambda} \ln ^{r} x_{-}\right) \circledast\left(x_{+}^{\mu} \ln ^{s-1} x_{+}\right)$exists and

$$
\left(x_{-}^{\lambda} \ln ^{r} x_{-}\right) \circledast\left(x_{+}^{\mu} \ln ^{s-1} x_{+}\right)=D_{\lambda}^{r} D_{\mu}^{s-1} g_{\lambda, \mu}(x)
$$

for some $s \in \mathbf{N}_{0}$. Since the neutrix convolution products in (28) and (29) exist, it follows that also $\left(x_{-}^{\lambda} \ln ^{r} x_{-}\right) \circledast\left(x_{+}^{\mu} \ln ^{s} x_{+}\right)$exists and

$$
\begin{aligned}
& \bar{\mu}\left(x_{-}^{\lambda} \ln ^{r} x_{-}\right) \circledast\left(x_{+}^{\mu} \ln ^{s} x_{+}\right)= \\
& =\left(x_{-}^{\lambda} \ln ^{r} x_{-}\right) \circledast\left[\bar{\mu} x_{+}^{\mu} \ln ^{s} x_{+}+s x_{+}^{\mu} \ln ^{s-1} x_{+}\right]-s\left(x_{-}^{\lambda} \ln ^{r} x_{-}\right) \circledast\left(x_{+}^{\mu} \ln ^{s-1} x_{+}\right)= \\
& =D_{\lambda}^{r} D_{\mu}^{s}\left[(\lambda+\mu+2) f_{\lambda, \mu}(x)\right]-s D_{\lambda}^{r} D_{\mu}^{s-1} g_{\lambda, \mu}(x)=\bar{\mu} D_{\lambda}^{r} D_{\mu}^{s} g_{\lambda, \mu}(x) .
\end{aligned}
$$

Since $\bar{\mu} \neq 0$, it follows by induction with respect to $s$ that our assertion holds for $(\lambda, \mu) \in$ $X_{k+1}$ and this completes the proof of Part II. 


\section{Part III: the general case.}

Denote

$$
Y_{i}:=\left\{(\lambda, \mu): \lambda>-i, \lambda \notin \mathbf{N}_{i-1}^{-}, \mu \notin-\mathbf{N} ; \lambda+\mu \notin \mathbf{Z}\right\} .
$$

We have to prove the assertion of the theorem for $(\lambda, \mu) \in Y_{i}$ and all $i \in \mathbf{N}$.

Evidently, the assertion is true for $(\lambda, \mu) \in Y_{1}$, due to Part II. Assume that the assertion holds for all pairs in $Y_{k}, k \in \mathbf{N}$ and let $(\lambda, \mu) \in Y_{k+1}$. Clearly, $(\bar{\lambda}, \mu) \in X_{k}$ and $\bar{\lambda} \neq 0$, where $\bar{\lambda}:=\lambda+1$.

Since the convolution product $\left(x_{-}^{\lambda+1} \ln ^{r} x_{-}\right)_{n} *\left(x_{+}^{\mu} \ln ^{s} x_{+}\right)$exists in the sense of Definition 1 for $n \in \mathbf{N}$, equations (2) can be used. Given an arbitrary $\phi \in \mathcal{D}$ (let the support of $\phi$ be contained in the interval $[a, b]$ ), we have

$$
\begin{aligned}
& \left\langle\left[\left(x_{-}^{\bar{\lambda}} \ln ^{r} x_{-}\right)_{n} *\left(x_{+}^{\mu} \ln ^{s} x_{+}\right)\right]^{\prime}, \phi(x)\right)=-\left\langle\left(x_{-}^{\bar{\lambda}} \ln ^{r} x_{-}\right)_{n} *\left(x_{+}^{\mu} \ln ^{s} x_{+}\right), \phi^{\prime}(x)\right\rangle= \\
& =\left\langle\left[(\bar{\lambda})\left(x_{-}^{\lambda} \ln ^{r} x_{-}+r\left(x_{-}^{\lambda} \ln ^{r-1} x_{-}\right]_{n} *\left(x_{+}^{\mu} \ln ^{s} x_{+}\right), \phi(x)\right\rangle+\right.\right. \\
& +\left\langle\left[x_{-}^{\bar{\lambda}} \ln ^{r} x_{-} \tau_{n}^{\prime}(x)\right] *\left(x_{+}^{\mu} \ln ^{s} x_{+}\right), \phi(x)\right\rangle .
\end{aligned}
$$

The support of $\tau_{n}^{\prime}(x)$ is contained in the interval $\left[-n-n^{-n},-n\right]$. Therefore, for $n>-a$,

$$
\left\langle\left(x_{-}^{\bar{\lambda}} \ln ^{\mathrm{r}} x_{-} \tau_{n}^{\prime}(x)\right) *\left(x_{+}^{\mu} \ln ^{s} x_{+}\right), \phi(x)\right\rangle=\int_{a}^{b} \phi(x) I_{n}(x) d x,
$$

where

$$
I_{n}(x):=\int_{-n-n^{-n}}^{-n}(-y)^{\bar{\lambda}} \ln ^{r}(-y) \tau_{n}^{\prime}(y)(x-y)^{\mu} \ln ^{s}(x-y) d y d x,
$$

with the functions $(-y)^{\bar{\lambda}} \ln ^{\gamma}(-y)$ and $(x-y)^{\mu} \ln ^{s}(x-y)$ integrable on the domain of integration. Integration by parts yields

$$
I_{n}(x)=h_{n}(x)+\int_{-n-n^{-n}}^{-n}\left[(-y)^{\bar{\lambda}} \ln ^{r}(-y)(x-y)^{\mu} \ln ^{s}(x-y)\right]^{\prime} \tau_{n}(y) d y,
$$

where

$$
h_{n}(x):=n^{\bar{\lambda}} \ln ^{r} n(x+n)^{\mu} \ln ^{s}(x+n) .
$$

Choosing a positive integer $p$ greater than $\lambda+\mu$, we can put

$$
h_{n}(x)=n^{\lambda+\mu+1} \sum_{i=0}^{p-1} \sum_{j=0}^{s} \frac{a_{i j} x^{i} \ln ^{j} n}{n^{i}}+O\left(n^{\lambda+\mu+1-p} \ln ^{r+s} n\right) .
$$

Since $\lambda+\mu$ is not an integer, we conclude that

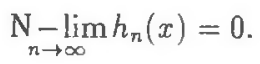

It is easily seen that

$$
\lim _{n \rightarrow \infty} \int_{-n-n^{-n}}^{-n}\left[(-y)^{\bar{\lambda}} \ln ^{r}(-y)(x-y)^{\mu} \ln ^{s}(x-y)\right]^{\prime} \tau_{n}(y) d y=0
$$


and thus, by (31), (32), (33) and (34),

$$
\underset{n \rightarrow \infty}{\mathrm{N}-\lim _{n}}\left\langle\left(x_{-}^{\bar{\lambda}} \ln ^{r} x_{-} \tau_{n}^{\prime}(x)\right) *\left(x_{+}^{\mu} \ln ^{s} x_{+}\right), \phi(x)\right\rangle=0 .
$$

Now, using (30), (35), the induction hypothesis and the fact that $\bar{\lambda} \neq 0$, it can be proved by induction with respect to $r$ that the assertion of the theorem is true for $(\lambda, \mu) \epsilon$ $Y_{k+1}$, in much the same way as in Part II (the role of $\mu$ in Part II is now played by $\lambda$ ).

By induction, the assertion in the general case follows.

Corollary. The neutrix convolution product $\left(x_{+}^{\lambda} \ln ^{r} x_{+}\right) \circledast\left(x_{-}^{\mu} \ln ^{3} x_{-}\right)$exists and

$$
\begin{aligned}
& \left(x_{+}^{\lambda} \ln ^{r} x_{+}\right) \circledast\left(x_{-}^{\mu} \ln ^{s} x_{-}\right)= \\
& =D_{\lambda}^{r} D_{\mu}^{s}\left[B(-\lambda-\mu-1, \mu+1) x_{+}^{\lambda+\mu+1}+B(-\lambda-\mu-1, \lambda+1) x_{-}^{\lambda+\mu+1}\right]
\end{aligned}
$$

for $r, s \in \mathbf{N}_{0}$ and $\lambda, \mu \in \mathbf{R} \backslash(-\mathbf{N})$ such that $\lambda+\mu \notin \mathbf{Z}$.

Proof. The assertion of the corollary follows immediately by the second part of Theorem 2, i.e. on replacing $x$ by $-x$ in equation (11).

The distributions $|x|^{\lambda} \ln ^{r}|x|$ and $\operatorname{sgn} x \cdot|x|^{\lambda} \ln ^{r}|x|$ are defined by

$$
|x|^{\lambda} \ln ^{r}|x|=x_{+}^{\lambda} \ln ^{r} x_{+}+x_{-}^{\lambda} \ln ^{r} x_{-}, \quad \operatorname{sgn} x \cdot|x|^{\lambda} \ln ^{r}|x|=x_{+}^{\lambda} \ln ^{r} x_{+}-x_{-}^{\lambda} \ln ^{r} x_{-} .
$$

We finally note that since the convolution products $\left(x_{+}^{\lambda} \ln ^{r} x_{+}\right) *\left(x_{+}^{\mu} \ln ^{3} x_{+}\right)$and $\left(x_{-}^{\lambda} \ln ^{r} x_{-}\right) *\left(x_{-}^{\mu} \ln ^{s} x_{-}\right)$exist by Definition 1 and since the neutrix convolution product is clearly distributive with respect to addition, it follows that further neutrix convolution products such as

$$
\begin{gathered}
\left(x_{-}^{\lambda} \ln ^{r} x_{-}\right) \circledast\left(|x|^{\mu} \ln ^{s}|x|\right), \quad\left(x_{+}^{\lambda} \ln ^{r} x_{+}\right) \circledast\left(|x|^{\mu} \ln ^{s}|x|\right), \\
\left(x_{-}^{\lambda} \ln ^{r} x_{-}\right) \circledast\left(\operatorname{sgn} x \cdot|x|^{\mu} \ln ^{s}|x|\right), \quad\left(|x|^{\lambda} \ln ^{r}|x|\right) \circledast\left(x_{-}^{\mu} \ln ^{s} x_{-}\right)
\end{gathered}
$$

exist for $r, s \in \mathbf{N}_{0}, \lambda, \mu \in \mathbf{R} \backslash(-\mathbf{N})$ with $\lambda+\mu \notin Z$. 


\section{References}

[1] J.G. van der Corput, Introduction to the neutrix calculus, J. Analyse Math. 7 (1959-60), 291-398.

[2] B. Fisher, Neutrices and the convolution of distributions, Univ. u Novom Sadu Zb. Rad. Prirod.-Mat. Fak. Ser. Mat. 17 (1987), 119-135.

[3] B. Fisher, A result on the neutrix convolution product of distributions, Publ. Math. Debrecen 37 (1990), 267-271.

[4] B. Fisher, On the neutrix convolution product $x^{s} \circledast x_{+}^{\lambda}$, in: Generalized Functions and Convergence (ed.: P. Antosik, A. Kaminski), World Scientific, Singapore 1990, 105-116.

[5] B. Fisher, The neutrix convolution product $x_{-}^{\lambda} \circledast x_{+}^{\mu}$, Dem. Math. 25 (1992), 525-532.

[6] B. Fisher, A. Kaminski, The neutrix convolution product $\ln x_{-} \circledast x_{+}^{r}$, Proc. of the Steklov Institute (in print).

[7] I.M. Gel'fand and G.E. Shilov, Generalized Functions, Vol. I, Academic Press 1964.

[8] E. Ozça-g, B. Fisher, On defining the distribution $x_{+}^{-r} \ln x_{+} x_{+}$, Rostock. Math. Kolloq. 42 (1990), 25-30.

[9] E. Özça-g and B. Fisher, On partial derivatives of the beta function, Rostock. Math. Kolloq. 45 (1991), 43-56.

B. Fisher and A. Kiliçman

Recenzent: Whadystaw Kierat

Department of Mathematics

University of Leicester

Leicester, LE1 7RH, England

\section{A. Kamiński}

Instytut Matematyki

Politechnika Ślaska

ul. Kaszubska 23

44-100 Gliwice 


\section{Streszczenie}

Splot $f * g$ dystrybucji Schwartza $f, g \in \mathcal{D}^{\prime}$ definiuje się wzorem

$$
\langle f * g, \phi\rangle=\left\langle f \tau_{n} * g, \phi\right\rangle,
$$

postulując, by granica istniala w przestrzeni $\mathcal{D}^{\prime}$ dla dowolnych funkcji $\phi \in \mathcal{D}$ oraz ciągów $\left(\tau_{n}\right)$ elementów przestrzeni $\mathcal{D}$ aproksymujących funkcję 1 i należących do określonej klasy.

Splot dystrybucji $f$ i $g$, oznaczany symbolem $f \circledast g$, rozumiany jest w tej pracy w ogólniejszym sensie, bowjem zakłada się, że granica po prawej stronie powyższej równości istnieje w sensie neutriksu (van der Corput [1]), wyznaczonego przez przestrzeń liniową funkcji zaniedbywalnych $f: \mathbf{N} \rightarrow \mathbf{R}$, generowaną przez wszystkie funkcje $f$ zbieżne do 0 oraz funkcje $f$ postaci:

$$
n^{\lambda} \ln ^{r-1} n, \ln ^{r} n \quad(\lambda>0, r \in \mathbf{N}) .
$$

Dowodzi się, że splot $f \circledast g$ istnieje w sensie neutriksu dla dystrybucji $f(x)=x_{-}^{\lambda} \ln ^{r} x_{-}$ i $g(x)=x_{+}^{\mu} \ln ^{3} x_{+}$oraz zachodzi równość:

$$
f \circledast g=D_{\lambda}^{r} D_{\mu}^{s}\left[B(-\lambda-\mu-1, \mu+1) x_{-}^{\lambda+\mu+1}+B(-\lambda-\mu-1, \lambda+1) x_{+}^{\lambda+\mu+1}\right]
$$

dla $r, s \in\{0\} \cup \mathbf{N}$ oraz $\lambda, \mu \in \mathbf{R} \backslash(-\mathbf{N})$, takich że $\lambda+\mu \notin \mathbf{Z}$, gdzie

$$
D_{\lambda}^{r}=\frac{\partial^{r}}{\partial \lambda^{r}}, \quad D_{\mu}^{s}=\frac{\partial^{s}}{\partial \mu^{s}},
$$

a $B$ oznacza funkcję beta Eulera.

Stąd wynika także istnienie następujących splotów:

$$
\begin{aligned}
& \left(x_{+}^{\lambda} \ln ^{r} x_{+}\right) \circledast\left(x_{-}^{\mu} \ln ^{s} x_{-}\right) ; \quad\left(x_{-}^{\lambda} \ln ^{r} x_{-}\right) \circledast\left(|x|^{\mu} \ln ^{s}|x|\right) \\
& \left(x_{+}^{\lambda} \ln ^{r} x_{+}\right) \circledast\left(|x|^{\mu} \ln ^{s}|x|\right) ; \quad\left(x_{-}^{\lambda} \ln ^{r} x_{-}\right) \circledast\left(\operatorname{sgn} x \cdot|x|^{\mu} \ln ^{s}|x|\right) ; \\
& \left(|x|^{\lambda} \ln ^{r}|x|\right) \circledast\left(x_{-}^{\mu} \ln ^{s} x_{-}\right)
\end{aligned}
$$

przy tych samych warunkach na $r, s, \lambda, \mu$, co poprzednio. 